Pacific Journal of Mathematics

THE INDEX OF CONVEXITY AND PARALLEL BODIES 


\title{
THE INDEX OF CONVEXITY AND PARALLEL BODIES
}

\author{
Gerald A. Beer
}

\begin{abstract}
Intuitively, the visibility function for a set $C$ in $R^{n}$ measures the $n$-dimensional volume of the star of a variable point of $C$. Suppose that the visibility function for $C$ is measurable. If the measure of $C$ is positive, normalizing the integral of the function produces a measure of the relative convexity of $C$, called the Index of convexity of $C$. The purpose of this paper is to study the relationship between the Index of convexity of a compact set $C$ in $R^{n}$ and the Indices of its parallel bodies. Continuity properties of the Index are established relative to an appropriate metric on the class of compact sets in $R^{n}$.
\end{abstract}

\section{Introduction.}

DEFINITION. The visibility function assigns to each point $x$ of a fixed measurable set $E$ in a Euclidean space $R^{n}$ the Lebesgue outer measure of $\{y: r x+(1-r) y \in E$ for each $r$ in $[0,1]\}$ and zero to each point of $R^{n} \backslash E$.

Definition. Let $E \subset R^{n}$ be a measurable set with measurable visibility function $v_{E}$, and suppose the Lebesgue measure of $E, m(E)$, is finite. If $m(E)>0$, the Index of convexity of $E, I(E)$, is given by $\int v_{E} /\left(m(E)^{2}\right) d m$. If $m(E)=0$, we agree to let $I(E)$ be 1 .

The reader will find a general treatment of the visibility function and the Index of convexity in [3]. Important for the present article are the following results: The visibility function for $E$ is uppersemicontinuous whenever $E$ is compact, and $I$ is upper-semicontinuous on the class of all compact sets in $R^{n}$ with an appropriate metric; namely, if $C$ and $K$ are compact sets, define $\bar{d}(C, K)$ to be sup $(d(C, K), m(C \Delta K))$ where $d$ denotes Hausdorff distance.

Let $B_{r}(x)$ denote the closed $r$-ball about a point $x$ in $R^{n}$.

Definition. Let $C$ be a compact set in $R^{n}$. The $\varepsilon$-parallel body of $C$, denoted by $B_{\varepsilon}(C)$, is the compact set $\bigcup_{x \in C} B_{\varepsilon}(x)$.

This paper illustrates the central role played by parallel bodies in the study of the Index of convexity. Using this concept, we can characterize those sequences $\left\{C_{k}\right\}$ of compact sets convergent in the $\bar{d}$ metric to a compact set $C$ of positive measure having the property that $I(C)=\lim _{k \rightarrow \infty} I\left(C_{k}\right)$.

We then consider the Index as a function of the radius $\varepsilon$ of the 
parallel body $B_{\varepsilon}(C)$ of a fixed compact set $C$ in $R^{n}$. This function is continuous if $C$ is starshaped. Also, if $C$ is contained in a flat of dimension $p$, its $p$ dimensional Index of convexity, if it is not "trivially" 1 , is determined by the Indices of its parallel bodies in $R^{n}$.

With a few exceptions we use the same terminology as in [1]. We denote ordinary Lebesgue measure by $m_{n}$ or simply by $m$ if only one space is under discussion. Conv ker $E$ and conv $E$ will indicate the convex kernel and convex hull of $E$, respectively. As usual, bd $E$, int $E$, and cl $E$ are the boundary, interior, and closure of $E$. Finally, $x y$ will denote the line segment joining $x$ to $y$. If $x \in C$, we say that $x$ sees $y$ via $C$ if $x y \subset C$. The star of $x$ relative to $C$ is simply all those points which $x$ sees via $C$. As alluded to above, $v_{C}$ represents the visibility function for a fixed set $C$, and $I(C)$ is the Index of $C$.

2. The characterization theorem. The visibility function $v_{C}$ of a compact set $C$ is continuous on $C$ if and only if the visibility functions for the parallel bodies of $C$, when restricted to $C$, converge uniformly to $v_{C}$ [2]. We establish here an analogous result for the unnormalized integral of the visibility function defined on a compact collection of compact sets in $R^{n}$ relative to the Hausdorff metric.

If $C$ is a compact set in $R^{n}$, denote the unnormalized integral of the visibilility function for $C, \int v_{C} d m$, by $V(C)$.

Lemma 1. Let $\left\{C_{l}\right\}$ be a collection of compact sets in $R^{n}$ convergent in the Hausdorff metric to a compact set $C$. Then $V(C) \geqq$ $\lim \sup _{l \rightarrow \infty} V\left(C_{l}\right)$.

Proof. If $x$ is in $C$, let $S(x)$ denote the star of $x$ relative to $C$, and let $S_{l}(x)$ denote star of $x$ relative to $C_{l}$. Since $\left\{C_{l}\right\}$ converges to $C$ in the Hausdorff metric, for any fixed $\varepsilon>0, B_{\varepsilon}(S(x))$ includes $S_{l}(x)$ for all sufficiently large integers $l$. This yields $v_{C}(x) \geqq \sup _{l \rightarrow \infty} v_{C_{l}}(x)$ for each $x$ in $C$. By Fatou's lemma

$$
\begin{aligned}
V(C) & =\int v_{C} d m \geqq \int_{C} \lim \sup _{l \rightarrow \infty} v_{C_{l}} d m \geqq \lim \sup _{l \rightarrow \infty} \int_{C} v_{C_{l}} d m \\
& =\lim \sup _{l \rightarrow \infty} \int v_{C_{l}} d m=\lim \sup _{l \rightarrow \infty} V\left(C_{l}\right)
\end{aligned}
$$

since $m\left(C_{l} \backslash C\right) \rightarrow 0$.

Hence, $V$ is an upper-semicontinuous function on the metric space of compact sets in $R^{n}$ with the Hausdorff metric. Since $\{x:\|x\| \leqq 1\}$ in $R^{n}$ is the Hausdorff limit of a sequence of compact sets of measure zero, $V$ fails to be globally continuous. 
In establishing our main result we use the following famous theorem of Dini: Let $\left\{f_{n}\right\}$ be a sequence of upper-semicontinuous nonnegative functions defined on a compact metric space $\mathscr{A}$. Suppose for each $x$ in $\mathscr{A},\left\{f_{n}(x)\right\}$ converges monotonically to zero. Then $\left\{f_{n}\right\}$ converges uniformly to the zero function on $\mathscr{A}$. For simplicity of notation, let $V_{k}(C)$ denote $V\left(B_{1 / k}(C)\right)$ for each $k \in Z^{+}$.

THEOREM 1. Let $\mathscr{A}$ be a compact collection of compact sets relative to the Hausdorff metric. The function $V: \mathscr{A} \rightarrow R$ is continuous on $\mathscr{A}$ if and only if $\left\{V_{k}\right\}$ converges uniformly to $V$ on $\mathscr{A}$ as $k \rightarrow \infty$.

Proof. Suppose first that $V$ is continuous on $\mathscr{A}$. The previous lemma implies that $\left\{V_{k}-V\right\}$ is a sequence of upper-semicontinuous functions. Clearly, the sequence converges monotonically to zero on $\mathscr{A}$. Since $\mathscr{A}$ is compact, Dini's theorem now applies.

Conversely, suppose $V$ is discontinuous at some compact set $C$ in $\mathscr{A}$. Since $V$ is upper-semicontinuous, there must then exist a sequence $\left\{C_{k}\right\}$ in $\mathscr{A}$ and an $\varepsilon>0$ satisfying $V(C)>V\left(C_{k}\right)+\varepsilon$ and $d\left(C_{k}, C\right)<1 / k$ for all $k$. By the definition of Hausdorff distance, $B_{1 / k}\left(C_{k}\right) \supset C$ so that $V_{k}\left(C_{k}\right)>V\left(C_{k}\right)+\varepsilon$. Thus, the convergence cannot be uniform on $\mathscr{A}$.

Suppose that $\left\{C_{l}\right\}$ is a sequence of compact sets in $R^{n}$ convergent to $C$ in the $\bar{d}$ metric (not merely in the Hausdorff metric), and $m(C)>0$. The following are necessary and sufficient conditions for $\lim _{l \rightarrow \infty} I\left(C_{l}\right)$ to exist and to equal $I(C)$.

THEOREM 2. Let $\left\{C_{l}\right\}$ be a collection of compact sets convergent in the metric $\bar{d}$ to a compact set $C$ of positive measure. Then $\lim _{l \rightarrow \infty} I\left(C_{l}\right)=I(C)$ if and only if $I\left(B_{1 / k}\left(C_{l}\right)\right) \rightarrow I\left(C_{l}\right)$ uniformly on $\left\{C_{l}: l \in Z^{+}\right\}$as $k \rightarrow \infty$.

Proof. Let $\mathscr{A}$ denote $\{C\} \cup\left\{C_{l}: l \in Z\right\}$. $\mathscr{A}$ is compact relative to the $\bar{d}$ metric and has only one limit point, the set $C$. Since Lebesgue measure is an upper-semicontinuous function on the metric space of compact sets in $R^{n}$ with the Hausdorff metric, an application of Dini's theorem yields the uniform convergence of $\left\{m\left(B_{1 / k}(F)\right)\right\}$ to $m(F)$ for each $F \in \mathscr{A}$ as $k \rightarrow \infty$. It follows that $\lim _{k \rightarrow \infty} m\left(B_{1 / k}\left(C_{k}\right)\right)=$ $m(C)$. A slight modification of the technique used in the preceding theorem now yields the sufficiency of the conditions as the Index of convexity is upper-semicontinuous with respect to the metric $\bar{d}$. Conversely, if $\lim _{l \rightarrow \infty} I\left(C_{l}\right)=I(C)$, then $\lim _{l \rightarrow \infty} V\left(C_{l}\right)=V(C)$. Our result now follows from Theorem 1 , as any uniformly convergent sequence of functions on $\mathscr{A}$ will automatically be uniformly convergent on 
$\left\{C_{l}: l \in Z^{+}\right\}$.

Since the Index of convexity is not upper-semicontinuous with respect to the Hausdorff metric [3], the necessity of using the $\bar{d}$ metric in the previous theorem is not surprising. To verify this, given $l \in Z^{+}$, let $C_{l}$ be the planar set $\{(x, y): 0 \leqq x \leqq 1,0 \leqq y \leqq 1\} \cup$ $\{(x, y): 1 \leqq x \leqq 2, y=k / l, k=0,1, \cdots l\}$. The sequence $\left\{C_{l}\right\}$ converges to $C=\{(x, y): 0 \leqq x \leqq 2,0 \leqq y \leqq 1\}$, and $I\left(C_{l}\right)=I(C)$ for all $l$. However, $I\left(B_{1 / k}\left(C_{l}\right)\right)$ does not converge uniformly to $I\left(C_{l}\right)$ on $\left\{C_{l}: l \in Z^{+}\right\}$. One can also see that both Theorems 1 and 2 fail if the metric only reflects convergence in measure.

3. Parallel bodies of a fixed set. Let $C$ be a fixed compact set in $R^{n}$. Define $I^{*}:(0, \infty) \rightarrow R$ by $I^{*}(r)=I\left(B_{r}(C)\right)$. It is obvious that $I^{*}(r)>0$ for $r>0$ and that $\lim _{r \rightarrow \infty} I^{*}(r)=1$.

Theorem 3. Let $C$ be a compact set in $R^{n}$. Then $I^{*}(r-) \leqq$ $I^{*}(r)=I^{*}(r+)$ for each $r>0$.

Proof. Let $\left\{s_{k}\right\}$ be a decreasing sequence of positive numbers convergent to $r$. The visibility function for $C$ is the pointwise limit of the sequence of visibility functions corresponding to $\left\{B_{s_{k}}(C)\right\}$. Hence $I^{*}(r)=I^{*}(r+)$. If $\left\{r_{k}\right\}$ is an increasing sequence of positive numbers convergent to $r$, then the visibility function for $\bigcup_{k=1}^{\infty} B_{r_{k}}(C)$ is the pointwise limit of the sequence of visibility function corresponding to $\left\{B_{r_{k}}(C)\right\}$. An application of the Dominated Convergence Theorem yields the existence of $I^{*}(r-)$. Since $\left\{B_{r_{k}}(C)\right\}$ converges in the $\bar{d}$ metric to $B_{r}(C)$, we have $I^{*}(r-) \leqq I^{*}(r)$.

A compact planar set for which $I^{*}$ has infinitely many discontinuities is

$$
(0,0) \cup \bigcup_{k=1}^{\infty}\left\{(x, y): \frac{1}{2 k+1} \leqq x^{2}+y^{2} \leqq \frac{1}{2 k}\right\}
$$

THeOREM 4. If $C$ is a compact starshaped set, $I^{*}$ corresponding to $C$ is continuous.

Proof. We need only show that $I^{*}$ is left continuous at each point of $(0, \infty)$. Fix $r$ in $(0, \infty)$, and let $\left\{r_{k}\right\}$ be an increasing sequence of positive reals convergent to $r$. As we have seen, $\left\{B_{r_{k}}(C)\right\}$ converges in the $\bar{d}$ metric to $B_{r}(C)$. Moreover, since $C$ is starshaped, $\bigcup_{k=1}^{\infty} B_{r_{k}}(C)$ is precisely int $B_{r}(C)$. The assertion now easily follows from the Dominated Convergence Theorem upon verifying that $v_{B_{r_{k}}(C)}(x) \rightarrow v_{B_{r}(C)}(x)$ for each $x$ in int $B_{r}(C)$.

Suppose such a point $x$ sees a point $z$ via int $B_{r}(C)$. The com- 
pactness of $x z$ forces the segment to be totally contained in $B_{r_{k}}(C)$ for $k$ sufficiently large. However, $x$ may see some points of $B_{r}(C)$ via line segments not wholly contained in the interior of $B_{r}(C)$. Fortunately, the arduous task of establishing that the set of such points has measure zero has been executed in [2] in proving that the visibility function for a compact starshaped set whose convex kernel has dimension exceeding $n-2$ is continuous on the interior of the set. It follows that almost every point in the star of $x$ relative to $B_{r}(C)$ is in the star of $x$ relative to $B_{r_{k}}(C)$ for $k$ sufficiently large so that $v_{B_{r_{k}}(C)}(x) \rightarrow v_{B_{r}(C)}(x)$.

A compact set that is the closure of an open set and that has Index 1 must be convex [3]. Hence, if $I^{*}(r)=1$ for some $r>0$, then $I^{*}(s)=1$ for all $s>r$.

Theorem 5. Let $C$ be a compact set in $R^{n}$. The number 1 is a value of $I^{*}$ corresponding to $C$ if and only if bd (conv $\left.C\right) \subset C$.

Proof. If bd (conv $C) \subset C$, it is easy to show that $B_{r}(C)$ is convex for sufficiently large $r$. Conversely, suppose bd (conv $C) \cap C^{c} \neq \varnothing$. Given a fixed $p$ in bd ( $\operatorname{conv} C) \cap C^{c}$, choose an outer unit normal $x$ to a hyperplane of support of $\operatorname{conv} C$ at $p$. Evidently, for each $r>0$, $p+r x \notin B_{r}(C)$. Since $p$ is a convex combination of points in $C$, say $\left\{x_{1}, x_{2}, \cdots, x_{k}\right\}$, the point $p+r x$ is a convex combination of $\left\{x_{1}+\right.$ $\left.r x, \cdots, x_{k}+r x\right\} \subset B_{r}(C)$. Hence $B_{r}(C)$ is never convex, so that $I^{*}(r)$ is never one.

Corollary. Let $C$ be a compact nonconvex starshaped set in $R^{n}$. Then $I^{*}$ corresponding to $C$ never assumes the value 1.

Even when $C$ is starshaped, it is not necessarily true that $I^{*}$ corresponding to $C$ be a monotone increasing function. It would be useful to characterize those compact sets having this property, for if $I^{*}$ were bounded, monotone increasing, and right continuous, a normalization of $I^{*}$ yields a probability distribution function.

If $\left\{C_{k}\right\}$ is a sequence of compact sets converging in the $\bar{d}$ metric to $C$, it may well occur that $C$ is contained in a hyperplane $H$. Relative to $H, C$ has an $n-1$ dimensional Index of convexity which we denote by $I^{H}(C)$. One might guess that if the projections of $\left\{C_{k}\right\}$ onto $H$ converged to $C$ in terms of $n-1$ dimensional measure, then $\lim \sup I\left(C_{k}\right) \leqq I^{H}(C)$ would follow. Unfortunately, the inequality is invalid. Let $H=\{(x, y, z): z=0\}$. Let $C_{k}=\left\{(x, y, z): x^{2}+\right.$ $\left.y^{2} \leqq 1, \quad 0 \leqq z \leqq 1 / k\right\} \cup\left\{(x, y, z):(x-2)^{2}+(y-2)^{2} \leqq 1, \quad 0 \leqq z \leqq 2 / k\right\}$. If $C=C_{1} \cap H$, then $\left\{C_{k}\right\} \rightarrow C$. However, $I^{H}(C)=1 / 2$ while $I\left(C_{k}\right)=5 / 9$ for all $k$. Clearly the projection of each $C_{k}$ is just $C$. 
We now show that the $n-1$ dimensional Index of such a set $C$ is the limit of the Indices of its parallel bodies in $R^{n}$.

THeORem 6. Let $H$ be a hyperplane in $R^{n}$. Suppose $C$ is a compact subset of $H$ and $m_{n-1}(C)>0$. Then $\lim _{k \rightarrow \infty} I\left(B_{1 / k}(C)\right)=I^{H}(C)$, the Index of convexity of $C$ with respect to $H$.

Proof. We may assume that $H=\left\{\left(x_{1}, \cdots, x_{n}\right): x_{n}=0\right\}$. Let $\pi: R^{n} \rightarrow R^{n-1}$ be defined by $\pi\left(x_{1}, \cdots, x_{n}\right)=\left(x_{1}, \cdots, x_{n-1}\right)$. Two facts are obvious: For each $k \in Z^{+}$we have $B_{1 / k}(C) \supset \pi(C) \times[-1 / k, 1 / k]$, and if $x$ belongs to $\pi(C) \times[-1 / k, 1 / k]$ then the star of $x$ relative to $B_{1 / k}(C)$ includes the Cartesian product of the star of $\pi(x)$ relative to $\pi(C)$ with $[-1 / k, 1 / k]$.

Suppose $p \in R^{n-1}$ does not belong to the star of $\pi(x)$ relative to $\pi(C)$. If $k$ is sufficiently large, no point of $\{p\} \times[-1 / k, 1 / k]$ can be seen by any point in $\{\pi(x)\} \times[-1 / k, 1 / k]$ via $B_{1 / k}(C)$ or else $\pi(x) p \subset \pi(C)$ by the compactness of $C$.

For each $k \in Z^{+}$and $y$ in $\pi(C)$, let $r_{k}(y)$ be the $n-1$ dimensional measure of the projection onto $H$ of the union of the stars of all points in $\{y\} \times[-1 / k, 1 / k]$ relative to $B_{1 / k}(C)$ minus $v_{\pi(C)}(y)$. Thus for fixed $k$ if $T(y)$ denotes $\bigcup_{x \in\{y\} \times[-1 / k, 1 / k]}\left\{z: x z \subset B_{1 / k}(C)\right\}$ we have

$$
r_{k}(y)=m_{n-1}(\pi(T(y)))-v_{\pi(C)}(y) .
$$

The compactness of $[-1 / k, 1 / k]$ and $B_{1 / k}(C)$ imply (i) $T(y)$ is compact so that $\pi(T(y))$ is compact for each $y \in \pi(C)$ (ii) if $\left\{y_{i}\right\}$ is a sequence in $\pi(C)$ with limit $y$, then

$$
\pi(T(y)) \supset \bigcap_{l=1}^{\infty} \bigcup_{i=l}^{\infty} \pi\left(T\left(y_{i}\right)\right) .
$$

By (1) and (2), $r$ is the difference of two upper-semicontinuous functions. Evidently $\lim _{k \rightarrow \infty} r_{k}(y)=0$ for each $y$ in $\pi(C)$. Since $B_{1 / k}(C) \subset B_{1 / k}(\pi(C)) \times[-1 / k, 1 / k]$ we conclude that for each $x$ in $\pi(C) \times[-1 / k, 1 / k]$

$$
v_{B_{1 / k}(C)}(x) \leqq \frac{2}{k}\left(v_{\pi(C)} \circ \pi(x)+r_{k} \circ \pi(x)\right) .
$$

Choosing $M$ to be the $n-1$ dimensional measure of $\pi\left(B_{1}(C)\right)$ we also see that

$$
v_{\pi(C)} \circ \pi(x)+r_{k} \circ \pi(x) \leqq M
$$

for $x \in \pi(C) \times[-1 / k, 1 / k]$ and all $k \in Z^{+}$.

To show $\lim _{k \rightarrow \infty} I\left(B_{1 / k}(C)\right)=I^{H}(C)$, it suffices to show

$$
\lim _{k \rightarrow \infty} \frac{k}{2} m\left(B_{1 / k}(C)\right)=m_{n-1}(\pi(C))
$$


and

$$
\lim _{k \rightarrow \infty} \frac{k^{2}}{4} \int v_{B_{1 / k}(C)} d x_{1} \cdots d x_{n}=\int v_{\pi(C)} d x_{1} \cdots d x_{n-1} .
$$

To establish (5) we again use the fact that $\pi(C) \times[-1 / k, 1 / k] \subset$ $B_{1 / k}(C) \subset B_{1 / k}(\pi(C)) \times[-1 / k, 1 / k]$ to conclude

$$
\frac{2}{k} m_{n-1}(\pi(C)) \leqq m_{n}\left(B_{1 / k}(C)\right) \leqq \frac{2}{k} m_{n-1}\left(B_{1 / k}(\pi(C))\right) .
$$

The compactness of $\pi(C)$ implies that $m_{n-1}\left(B_{1 / k}(\pi(C))\right) \rightarrow m_{n-1}(\pi(C))$ as $k \rightarrow \infty$ and (5) easily follows.

Recalling that the star of each $x$ in $\pi(C) \times[-1 / k, 1 / k]$ relative to $B_{1 / k}(C)$ includes the Cartesian product of the star of $\pi(x)$ relative to $\pi(C)$ with $[-1 / k, 1 / k]$, we have

$$
\begin{aligned}
& \frac{k^{2}}{4} \int v_{B_{1 / k}(C)} d x_{1} \cdots d x_{n} \geqq \frac{k^{2}}{4} \int_{\pi(C)}\left(\int_{-1 / k}^{1 / k} v_{B_{1 / k}(C)} d x_{n}\right) d x_{1} \cdots d x_{n-1} \\
\geqq & \frac{k^{2}}{4} \int_{\pi(C)}\left(\int_{-1 / k}^{1 / k} \frac{2}{k} v_{\pi(C)} \circ \pi d x_{n}\right) d x_{1} \cdots d x_{n-1} \\
= & \frac{k^{2}}{4} \int_{\pi(C)} v_{\pi(C)} \cdot \frac{4}{k^{2}} d x_{1} \cdots d x_{n-1}=\int v_{\pi(C)} d x_{1} \cdots d x_{n-1}
\end{aligned}
$$

since $v_{\pi(C))} \circ \pi$ is constant on any vertical line.

The reverse inequality does not follow so easily. We make a preliminary decomposition:

$$
\begin{aligned}
& \frac{k^{2}}{4} \int v_{B_{1 / k}(C)} d x_{1} \cdots d x_{n} \\
= & \frac{k^{2}}{4} \int_{\pi(C)}\left(\int_{-1 / k}^{1 / k} v_{B_{1 / k}(C)} d x_{n}\right) d x_{1} \cdots d x_{n-1} \\
& +\frac{k^{2}}{4} \int_{B_{1 / k}(\pi(C)) / \pi(C)}\left(\int_{-1 / k}^{1 / k} v_{B_{1 / k}(C)} d x_{n}\right) d x_{1} \cdots d x_{n-1} .
\end{aligned}
$$

Since

$$
\frac{k^{2}}{4} \int_{-1 / k}^{1 / k} v_{B_{1 / k}(C)} d x_{n} \leqq M
$$

for all $k$, the second integral in (7) can be made less than $\varepsilon / 3$ if $k$ is sufficiently large.

Since $r_{k}$ is measurable for each $k$, by Egoroff's theorem there is a subset $F$ of $\pi(C)$ such that $m_{n-1}(F)<\varepsilon / 3 M$ and $r_{k}$ converges to zero uniformly on $\pi(C) / F$. For any $k \in Z^{+}$, we now have using (8)

$$
\frac{k^{2}}{4} \int_{F}\left(\int_{-1 / k}^{1 / k} v_{B_{1 / k}(C)} d x_{n}\right) d x_{1} \cdots d x_{n-1}<\frac{\varepsilon}{3 M} \cdot M=\frac{\varepsilon}{3} .
$$


Pick $k$ so large that $\left|r_{k}(y)\right|<\varepsilon / 3 M$ uniformly on $\pi(C) / F$. We can now conclude from (3) and (4) that

$$
\begin{aligned}
& \frac{k^{2}}{4} \int_{\pi(C) / F}\left(\int_{-1 / k}^{1 / k} v_{B_{1 / k}(C)} d x_{n}\right) d x_{1} \cdots d x_{n-1} \\
\leqq & \frac{k^{2}}{4} \int_{\pi(C) / F}\left(\int_{-1 / k}^{1 / k} \frac{2}{k}\left(v_{\pi(C)} \circ \pi+r_{k} \circ \pi\right) d x_{n}\right) d x_{1} \cdots d x_{n-1} \\
\leqq & \int_{\pi(C) / F} v_{\pi(C)}+\frac{\varepsilon}{3 M} d x_{1} \cdots d x_{n-1} \leqq \int_{\pi(C)} v_{\pi(C)} d x_{1} \cdots d x_{n-1}+\frac{\varepsilon}{3} .
\end{aligned}
$$

Combining our three integrals over $B_{1 / k}(\pi(C)) / \pi(C), F$ and $\pi(C) / F$ we have

$$
\frac{k^{2}}{4} \int v_{B_{1 / k}(C)} d x_{1} \cdots d x_{n} \leqq \int v_{\pi(C)} d x_{1} \cdots d x_{n-1}+\varepsilon
$$

when $k$ is sufficiently large so that (6) is established.

Theorem 6 has the following obvious generalization.

THEOREM 7. Let $C$ be a compact set in $R^{n}$ contained in a flat $F$ of dimension $p$, and suppose $m_{p}(C)>0$. If $I^{F}(C)$ denotes the Index of convexity of $C$ with respect to $F$, then $\lim _{k \rightarrow \infty} I\left(B_{1 / k}(C)\right)=I^{F}(C)$.

The proofs of Theorems 6 and 7 are identical modulo replacing $[-1 / k, 1 / k]$ by an $n-p$ dimensional sphere of radius $1 / k$. Of course, the notation required to establish Theorem 3 would be horrendous, and for this reason we confined ourselves to $p=n-1$.

Can we define the Index of convexity of a compact $C$ of measure zero to be the limit of the Indices of its parallel bodies? If $C$ is a compact set of positive measure, then $\lim _{r \rightarrow 0^{+}} I^{*}(r)=I(C)$, but if $m(C)=0$, then the limit need not even exist. To see that $I^{*}$ may behave poorly, we construct a peculiar compact set $C$ in the plane consisting of a countable collection of vertical segments. Each segment has length one and lies in the strip $\{(x, y): 0 \leqq y \leqq 1\}$. After placing a segment at the origin, we construct the others in an iterative manner. Let $\delta_{1}=1 / 8$. Initially place two vertical segments at $x=1-\delta_{1}$ and at $x=1+\delta_{1}$. Adjoin to this set 4 equally spaced segments between $x=1 / 4 \delta_{1}$ and $x=3 / 4 \delta_{1}$. Denote half the distance between such segments by $\delta_{2}$. Having chosen $\delta_{1}, \delta_{2}, \cdots, \delta_{n}$, construct $2^{2^{n}}$ equally spaced vertical segments between $x=1 / 4 \delta_{n}$ and $x=3 / 4 \delta_{n}$, and denote half the distance between adjacent new segments by $\delta_{n+1}$. Let $C$ be the union of the segments so constructed. Although $I^{*}\left(\delta_{n}-\right)<1 / 2$ for all $n$, $\lim _{n \rightarrow \infty} I^{*}\left(\delta_{n}\right)=1$. This example also indicates that $I^{*}$ need not be of bounded variation. 


\section{REFERENCES}

1. G. A. Beer, Continuity Properties of the Visibility Function, to appear, Michigan Math. J., 20 (1973), 297-302.

2. - The continunity of the visibility function on a starshaped set, Canad. J. Math., 24 (1972), 989-992.

3. - The index of convexity and the visibility function, Pacific J. Math., 44 (1973), 59-67.

4. F. A. Valentine, Convex Sets, McGraw-Hill, New York, 1964.

Received May 7, 1973 and in revised form November 25, 1973.

California State University 



\section{PACIFIC JOURNAL OF MATHEMATICS}

\section{EDITORS}

RICHARD ARENS (Managing Editor)

University of California

Los Angeles, California 90024

\section{J. DUGUNDJI}

Department of Mathematics University of Southern California Los Angeles, California 90007

D. Gilbarg and J. Milgram

Stanford University

Stanford, California 94305

University of Washington
Seattle, Washington 98105

ASSOCIATE EDITORS
E. F, BECKENBACH
B. H. NEUMANN
F. WOLF
K. Yoshida

\section{SUPPORTING INSTITUTIONS}

\author{
UNIVERSITY OF BRITISH COLUMBIA \\ CALIFORNIA INSTITUTE OF TECHNOLOGY \\ UNIVERSITY OF CALIFORNIA \\ MONTANA STATE UNIVERSITY \\ UNIVERSITY OF NEVADA \\ NEW MEXICO STATE UNIVERSITY \\ OREGON STATE UNIVERSITY \\ UNIVERSITY OF OREGON \\ OSAKA UNIVERSITY
}

\author{
UNIVERSITY OF SOUTHERN CALIFORNIA \\ STANFORD UNIVERSITY \\ UNIVERSITY OF TOKYO \\ UNIVERSITY OF UTAH \\ WASHINGTON STATE UNIVERSITY \\ UNIVERSITY OF WASHINGTON \\ * * * * \\ AMERICAN MATHEMATICAL SOCIETY \\ NAVAL WEAPONS CENTER
}

The Supporting Institutions listed above contribute to the cost of publication of this Journal, but they are not owners or publishers and have no responsibility for its content or policies.

Mathematical papers intended for publication in the Pacific Journal of Mathematics should be in typed form or offset-reproduced, (not dittoed), double spaced with large margins. Underline Greek letters in red, German in green, and script in blue. The first paragraph or two must be capable of being used separately as a synopsis of the entire paper. Items of the bibliography should not be cited there unless absolutely necessary, in which case they must be identified by author and Journal, rather than by item number. Manuscripts, in duplicate if possible, may be sent to any one of the four editors. Please classify according to the scheme of Math. Rev. Index to Vol. 39. All other communications to the editors should be addressed to the managing editor, or Elaine Barth, University of California, Los Angeles, California, 90024.

100 reprints are provided free for each article, only if page charges have been substantially paid. Additional copies may be obtained at cost in multiples of 50 .

The Pacific of Journal Mathematics is issued monthly as of January 1966. Regular subscription rate: $\$ 72.00$ a year (6 Vols., 12 issues). Special rate: $\$ 36.00$ a year to individual members of supporting institutions.

Subscriptions, orders for back numbers, and changes of address should be sent to Pacific Journal of Mathematics, 103 Highland Boulevard, Berkeley, California, 94708.

PUBLISHED BY PACIFIC JOURNAL OF MATHEMATICS, A NON-PROFIT CORPORATION

Printed at Kokusai Bunken Insatsusha (International Academic Printing Co., Ltd.), 270, 3-chome Totsuka-cho, Shinjuku-ku, Tokyo 160, Japan.

Copyright (C) 1973 by Pacific Journal of Mathematics Manufactured and first issued in Japan 


\section{Pacific Journal of Mathematics}

\section{Vol. 53, No. $2 \quad$ April, 1974}

Kenneth Abernethy, On characterizing certain classses of first countable spaces by



Ross A. Beaumont and Donald Lawver, Strongly semisimple abelian groups .......

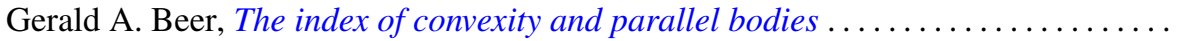

Victor P. Camillo and Kent Ralph Fuller, On Loewy length of rings ..............

Stephen LaVern Campbell, Linear operators for which $T^{*} T$ and $T T^{*}$ commute.

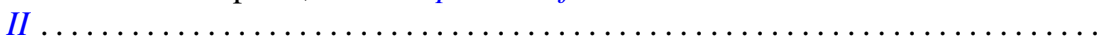

Charles Kam-Tai Chui and Philip Wesley Smith, Characterization of a function by



Allan L. Edelson, Conjugations on stably almost complex manifolds . ...........

Patrick John Fleury, Hollow modules and local endomorphism rings . . ..........

Jack Tilden Goodykoontz, Jr., Connectedness im kleinen and local connectedness in

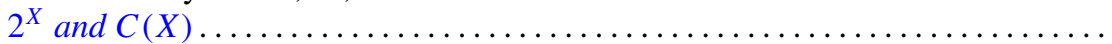

Robert Edward Jamison, II, Functional representation of algebraic intervals .......

Athanassios G. Kartsatos, Nonzero solutions to boundary value problems for



Soon-Kyu Kim, Dennis McGavran and Jingyal Pak, Torus group actions on simply



David Anthony Klarner and R. Rado, Arithmetic properties of certain recursively

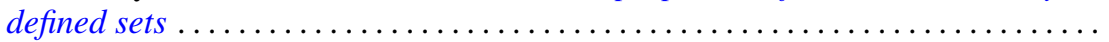

Ray Alden Kunze, On the Frobenius reciprocity theorem for square-integrable



John Lagnese, Existence, uniqueness and limiting behavior of solutions of a class of differential equations in Banach space...

Teck Cheong Lim, A fixed point theorem for families on nonexpansive mappings Lewis Lum, A quasi order characterization of smooth continua

Andy R. Magid, Principal homogeneous spaces and Galois extensions . .

Charles Alan McCarthy, The norm of a certain derivation ..... . .

Louise Elizabeth Moser, On the impossibility of obtaining $S^{2} \times S^{1}$ by elementary surgery along a knot. .

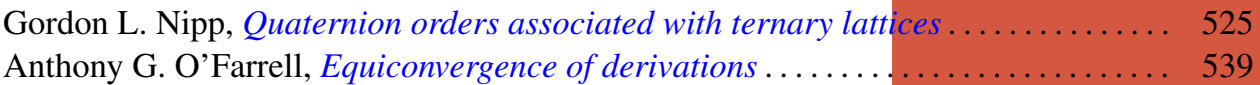

Dorte Olesen, Derivations of $A W^{*}$-algebras are inner . . . . . . . . . . . . . . . 555

Dorte Olesen and Gert Kjærgaard Pedersen, Derivations of $C^{*}$-algebras have

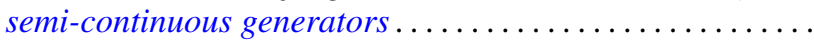

Duane O’Neill, On conjugation cobordism.

Chull Park and S. R. Paranjape, Probabilities of Wiener paths crossing differentiable

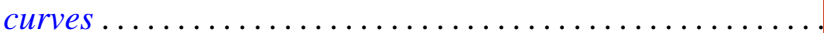

Edward Ralph Rozema, Almost Chebyshev subspaces of $L^{1}(\mu$;

Lesley Millman Sibner and Robert Jules Sibner, A note on the Atiyah-Bott fixed

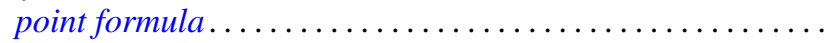

Betty Salzberg Stark, Irreducible subgroups of orthogonal groups generated by

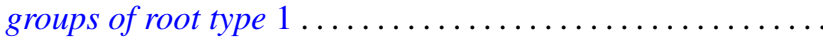

N. Stavrakas, A note on starshaped sets, $(k)$-extreme points and the half ray

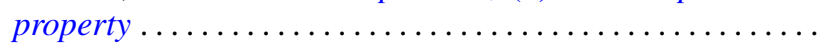

Carl E. Swenson, Direct sum subset decompositions of $Z \ldots \ldots$ 